\title{
Electroweak constraints on new physics
}

\author{
F. del Aguila ${ }^{a *}$ and $\mathbf{J}$. de Blas ${ }^{b * *}$ \\ ${ }^{a}$ CAFPE and Depto. de Física Teórica y del Cosmos, Universidad de Granada, E-18071 Granada, Spain \\ ${ }^{b}$ Department of Physics, University of Notre Dame, Notre Dame, Indiana 46556, USA
}

Received XXXX, revised XXXX, accepted XXXX

Published online XXXX

Key words Effective Lagrangian, electroweak precision data constraints, Higgs limits, extended models. Subject classification 12.15.-y, 12.60.Cn, 13.66.Jn, 14.60.St, 14.80.Bn

\begin{abstract}
We briefly review the limits on new interactions implied by electroweak precision data. Special attention is payed to the bounds on the Higgs boson mass. We also comment on the required cancellation among the new contributions to precisely measured electroweak observables in any Standard Model extension, if the new particles have to evade the indirect constraints on their couplings and masses but still remain at the LHC reach.
\end{abstract}

Copyright line will be provided by the publisher

\section{Introduction}

The time for establishing the mechanism of the Standard Model (SM) symmetry breaking seems to have arrived with the large hadron collider (LHC) era. Thus, it is widely believed that the SM is a low energy effective theory and that new physics must exist near the TeV scale which makes natural the observed values of the gauge boson and fermion masses. However, the four scenarios with or without the SM Higgs and/or new physics observed at LHC are still possible. Although it shall be paradoxical that electroweak precision data (EWPD) are in agreement with the SM predictions at the few per mille level [1,2], implying that the new physics scale is relatively large, but new resonances other than the SM Higgs are detected at the LHC [3]. EWPD also disfavor a SM Higgs mass much larger than its present direct limit and that no new physics is found up to a few TeV [4] (see below). At any rate, EWPD and direct searches are complementary and whatever physics LHC reveals, it shall fulfill the indirect constraints.

Physics could be unexpected but we shall assume that we will be finally left with the SM plus some new particles with masses above the electroweak scale. Such a scenario can be described for energies below a few hundreds of $\mathrm{GeV}$ by an effective Lagrangian with the SM fields and gauge symmetry. The new physics being encoded in the operators of dimension $d>4$. In the following we update the limits on these operators, assuming universality and taking one at a time. In general, only those contributing to observables showing (small) deviations from the SM predictions are not suppressed at the per cent level. We then explain how to accommodate a large Higgs mass, which is the only SM parameter still unknown. What can be done invoking new heavy neutrinos and/or vector bosons. Finally, we comment on how to fulfill the EWPD constraints and still allow for new resonances at the LHC reach.

\section{Effective Lagrangian approach, EWPD and model independent limits}

Let us write the effective Lagrangian with the SM fields and symmetries

$$
\mathcal{L}_{\text {eff }}=\sum_{d=4}^{\infty} \frac{1}{\Lambda^{d-4}} \mathcal{L}_{d}=\mathcal{L}_{4}+\frac{1}{\Lambda} \mathcal{L}_{5}+\frac{1}{\Lambda^{2}} \mathcal{L}_{6}+\ldots, \quad \mathcal{L}_{d}=\sum_{i} \alpha_{i}^{d} \mathcal{O}_{i}^{d}, \quad\left[\mathcal{O}_{i}^{d}\right]=d,
$$

\footnotetext{
*E-mail: faguila@ugr.es

** E-mail: jdeblasm@nd.edu
} 


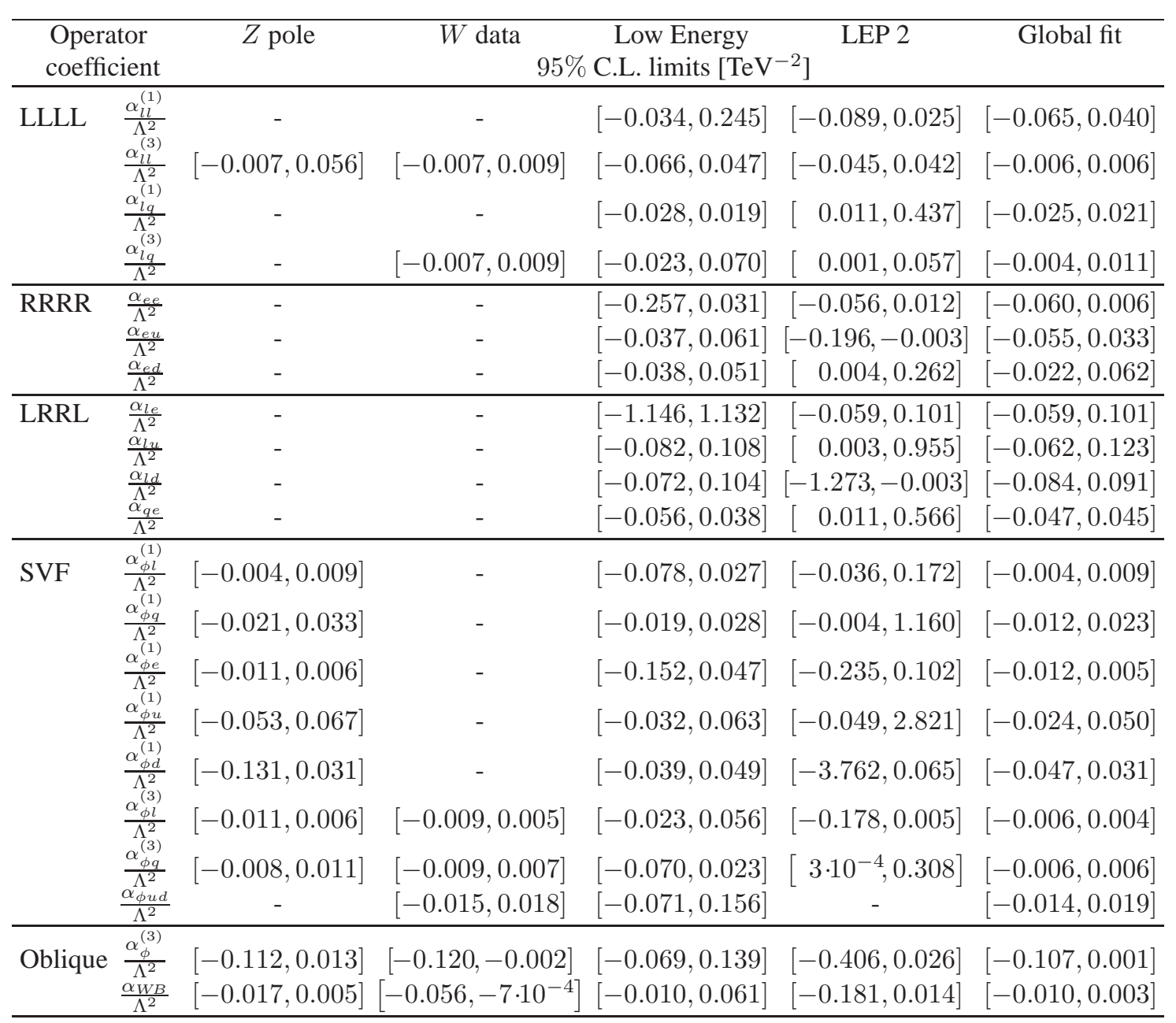

Table $195 \%$ C.L. limits on (90\% confidence interval of) the dimension six operator coefficients entering in EWPD. The limits are obtained from a fit considering only one operator at a time and for each data set. Limits are in units of $\mathrm{TeV}^{-2}$. The different columns show the results for different fits depending on the observables included. The second column ( $W$ data) also includes the constraints from CKM universality. When a given operator contributes to a physical process from which any of the SM inputs is derived, it indirectly corrects the predictions for all electroweak observables (e.g., the operator $\mathcal{O}_{l l}^{(3)}$ which modifies the prediction for the muon decay constant $G_{\mu}$ ).

where $\Lambda$ is the (unknown) cutoff scale up to which the effective Lagrangian description is valid, and each $\mathcal{L}_{d}$ contains all the local operators of canonical mass dimension $d$ allowed by the symmetries. $\left(\mathcal{L}_{5}\right.$ only contains one operator [5,6], which violates lepton number and can be neglected because it is proportional to the very tiny neutrino masses and then plays no rôle in our analysis [7].) The operators of dimension six $\mathcal{O}_{i}$ are classified in [6] (see for a non-redundant set [8]). In the table we update the limits on their coefficients $\alpha_{i} / \Lambda^{2}$. The data included in the fit are described in [4] 9-12], but updated to their more recent values. We separate them in four sets and collect the corresponding bounds in the first four columns. The global fit to all data is gathered in the last column. We assume universality and the fits are performed adding one operator at a time to the SM.

As can be observed, some of the most significant departures ( $\sim 1 \sigma$ or larger) from the SM predictions can be eased with few of these operators. This translates into asymmetric intervals. For instance, the excess 


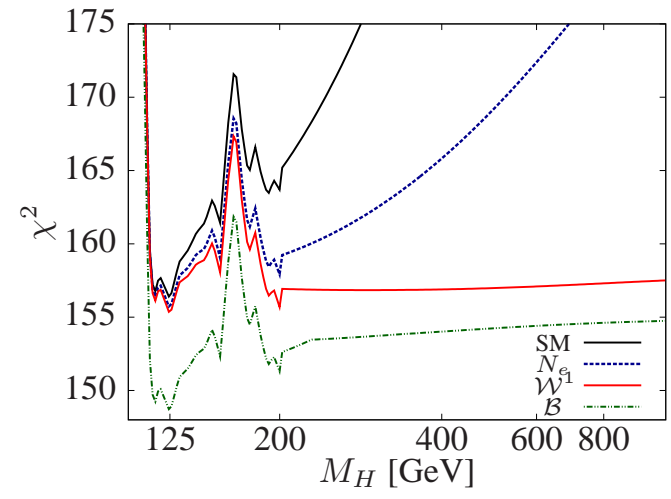

a)

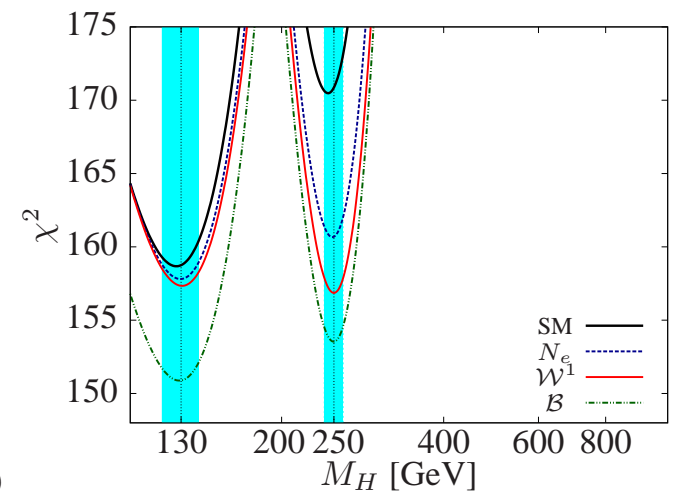

b)

Fig. 1 a) From top to bottom, minimum $\chi^{2}$ as a function of the Higgs mass for the SM fit, and the fits including besides a heavy neutrino singlet $N_{e}$ coupled to the first lepton family, a vector triplet of hyperchage one $\mathcal{W}^{1}$, and a neutral vector singlet $\mathcal{B}$ (see [4, 9]1] for conventions). b) The same but assuming that the SM Higgs is found to have a mass $M_{H}=130 \pm 10 \mathrm{GeV}$ or $M_{H}=250 \pm 10 \mathrm{GeV}$ (blue bands).

of the hadronic cross section observed at LEP 2 can be explained by four-fermion operators involving electrons and quarks, like $\mathcal{O}_{l q}^{(1,3)}$, etcetera. Parity violation in Møller scattering can be improved by $\mathcal{O}_{l l}^{(1)}$ or $\mathcal{O}_{e e}$, for example. On the other hand, the relatively large value of the $W$ mass can be accounted by $\mathcal{O}_{\phi}^{(3)}$ and $\mathcal{O}_{W B}$. While the large forward-backward bottom asymmetry results in an asymmetric $\alpha_{\phi d}^{(1)}$ interval, although we assume universality. At any rate, the size and asymmetry of the intervals get reduced when all data are considered.

\section{Implications on the Higgs mass}

In the previous fits to dimension six operators the SM parameters are fixed to their best value in the fit to the SM alone, except for the Higgs mass $M_{H}$ which is left free. This, in general, prefers to be next to its direct lower limit of $114 \mathrm{GeV}$ [1, 2, 13]. In the left figure we show the $\chi^{2}$ dependence on $M_{H}$ in the fit to the SM alone with all SM parameters free (upper black solid line). As it is apparent, if the SM Higgs is found to be relatively heavy, further physics has to cancel its one-loop contributions to the different electroweak precision observables, in order to restore the excellent agreement with the data. In particular, it has to balance the negative quantum correction to the $\rho=M_{W}^{2} / M_{Z}^{2} \cos ^{2} \theta_{W}$ parameter [14]. This can be done at tree level increasing the numerator or decreasing the denominator, yielding in both cases the required positive contribution. The former can be effectively achieved reducing the SM contribution to the Fermi constant $G_{\mu}$ by mixing the electron neutrino with a sterile heavy neutrino $N_{e}$ [9] 15], and the latter mixing the $Z^{0}$ boson with heavier extra vector bosons [11, 16]. 11 In the left figure we show the effect of both possibilities. The second upper line (blue dashed) corresponds to the heavy neutrino addition, which can not completely account for a heavy Higgs but improves the global fit. Whereas there are two gauge boson additions balancing the heavy Higgs corrections to EWPD, named $\mathcal{B}$ and $\mathcal{W}^{1}$ in [11], respectively (bottom green dotted-dashed and second bottom red solid lines in the figure). In these three fits the only SM parameters left free, besides the Higgs mass, are the strong coupling constant and the top mass. The large $\chi^{2}$ values on the ordinate reminds the large number (212) of data included in the fits. Finally, in the right figure we plot the same as in the left one but replacing the present large collider bounds [13] by two guesses of the Higgs mass eventually measured at CERN [17].

\footnotetext{
1 Note that in the operator basis chosen here corrections to $G_{\mu}$ can be encoded either in $\mathcal{O}_{\phi l}^{(3)}$ or $\mathcal{O}_{l l}^{(1,3)}$. While, direct corrections to the $\rho$ parameter are accounted by $\mathcal{O}_{\phi}^{(3)}$, allowing for large $M_{H}$ values for negative $\alpha_{\phi}^{(3)}$ (see Table 1 .
} 


\section{Cancelling contributions from extended spectra}

The previous fits make apparent the paradox that the SM describes physics up to the LEP 2 energy $(\sim$ $209 \mathrm{GeV}$ ) with a precision in general below the per cent level, but we still expect that LHC will discover further resonances near the $\mathrm{TeV}$ [3]. If so, a model dependent pattern of cancellations must arise resulting in small contributions to electroweak precision observables. The corresponding discussion for the case of extra gauge bosons is presented in [11, 12]. Examples with cancellations based on custodial symmetries can be found in [18] for extra quarks or in [19] for extra leptons. However, in these models flavor plays an essential rôle because the new fermions mainly mix with the third family, as may be in Nature.

Acknowledgements We are grateful to the Corfu Institute 2010 organizers for their habitual kind hospitality, and to M. Pérez-Victoria for collaboration in the work reviewed here. This work has been partially supported by MICINN (FPA2006-05294 and FPA2010-17915) and by Junta de Andalucía (FQM 101, FQM 3048 and FQM 6552). The work of J.B. has been supported in part by the U.S. National Science Foundation under Grant PHY-0905283-ARRA.

\section{References}

[1] K. Nakamura et al. [Particle Data Group], J. Phys. G 37, 075021 (2010).

[2] The ALEPH, CDF, D0, DELPHI, L3, OPAL, SLD Collaborations, the LEP Electroweak Working Group, the Tevatron Electroweak Working Group, and the SLD electroweak and heavy flavour groups, arXiv:1012.2367 [hep-ex]; M. Goebel on behalf of the Gfitter Group, PoS ICHEP2010, 570 (2010) arXiv:1012.1331] [hep-ph]].

[3] R. Barbieri and A. Strumia, arXiv:hep-ph/0007265

[4] J. de Blas, Ph.D.Thesis.

[5] S. Weinberg, Phys. Rev. Lett. 43, 1566 (1979).

[6] W. Buchmuller and D. Wyler, Nucl. Phys. B 268, 621 (1986); C. Arzt, M. B. Einhorn and J. Wudka, Nucl. Phys. B 433, 41 (1995) [arXiv:hep-ph/9405214.

[7] For reviews see: R. N. Mohapatra, et al., Rept. Prog. Phys. 70, 1757 (2007) |hep-ph/0510213|; M. Raidal, et al., Eur. Phys. J. C57, 13 (2008) |arXiv:0801.1826 [hep-ph]]. See also: A. Abada et al., JHEP 0712, 061 (2007) [arXiv:0707.4058 [hep-ph]]; F. del Aguila et al., Acta Phys. Polon. B 38, 3339 (2007) arXiv:0710.2923 [hep-ph]]; X. G. He et al., Phys. Rev. D 80, 073012 (2009) [arXiv:0907.1607][hep-ph]].

[8] B. Grzadkowski et al., Nucl. Phys. B 689, 108 (2004) [arXiv:hep-ph/0310159]; J. A. Aguilar-Saavedra, Nucl. Phys. B 812, 181 (2009) [arXiv:0811.3842 [hep-ph]]; Nucl. Phys. B 843, 638 (2011) |arXiv:1008.3562 [hepph]]; D. Nomura, JHEP 1002, 061 (2010) |arXiv:0911.1941 [hep-ph]].

[9] F. del Aguila, J. de Blas and M. Pérez-Victoria, Phys. Rev. D 78, 013010 (2008) [arXiv:0803.4008 [hep-ph]].

[10] F. del Aguila et al., Phys. Lett. B 683, 282 (2010) [arXiv:0911.3158 [hep-ph]]; F. del Aguila, J. A. AguilarSaavedra and J. de Blas, arXiv:1012.1327 [hep-ph].

[11] F. del Aguila, J. de Blas and M. Pérez-Victoria, JHEP 1009, 033 (2010) [arXiv:1005.3998 [hep-ph]].

[12] F. del Aguila et al., [arXiv:1104.5512 [hep-ph]].

[13] R. Barate et al. [LEP Working Group for Higgs boson searches and ALEPH Collaboration and and], Phys. Lett. B 565, 61 (2003) |arXiv:hep-ex/0306033]; The TEVNPH Working Group, arXiv:1007.4587 [hep-ex]; T. Aaltonen et al. [CDF and D0 Collaboration], arXiv:1103.3233 [hep-ex].

[14] M. E. Peskin and J. D. Wells, Phys. Rev. D 64, 093003 (2001) [arXiv:hep-ph/0101342].

[15] F. del Aguila, J.A. Aguilar-Saavedra and J. de Blas, Acta Phys. Polon. B40, 2901 (2009) arXiv:0910.2720 hepph]]; See for previous limits P. Langacker and D. London, Phys. Rev. D 38, 907 (1988); E. Nardi, E. Roulet and D. Tommasini, Phys. Lett. B 327, 319 (1994) |hep-ph/9402224|; S. Bergmann and A. Kagan, Nucl. Phys. B 538, 368 (1999) |hep-ph/9803305|; B. Bekman et al., Phys. Rev. D 66, 093004 (2002) |hep-ph/0207015|

[16] M. S. Chanowitz, arXiv:0806.0890 [hep-ph].

[17] T. Vickey [ATLAS Collaboration and CMS Collaboration], arXiv:0806.1330 [hep-ex]; E. Richter-Was [Atlas Collaboration], Acta Phys. Polon. B 40, 1909 (2009) [arXiv:0903.4198 [hep-ex]].

[18] K. Agashe et al., Phys. Lett. B 641, 62 (2006) |arXiv:hep-ph/0605341]; M. S. Carena et al., Nucl. Phys. B 759, 202 (2006) |arXiv:hep-ph/0607106]; Phys. Rev. D 76, 035006 (2007) [arXiv:hep-ph/0701055]; A. Atre et al., Phys. Rev. D 79, 054018 (2009) [arXiv:0806.3966][hep-ph]]; A. Atre et al., arXiv:1102.1987[hep-ph]; see also F. del Aguila, M. Pérez-Victoria and J. Santiago, Phys. Lett. B 492, 98 (2000) |arXiv:hep-ph/0007160]; JHEP 0009, 011 (2000) [arXiv:hep-ph/0007316].

[19] K. Agashe, Phys. Rev. D 80, 115020 (2009) [arXiv:0902.2400 [hep-ph]]; F. del Aguila, A. Carmona and J. Santiago, JHEP 1008, 127 (2010) arXiv:1001.5151 [hep-ph]]; Phys. Lett. B 695, 449 (2011) arXiv:1007.4206 [hep-ph]]. 